\title{
Weak photonic band gap effect on the fluorescence lifetime in three-dimensional colloidal photonic crystals
}

\author{
Zhi-Yuan Li and Zhao-Qing Zhang \\ Department of Physics, Hong Kong University of Science and Technology, Clear Water Bay, Kowloon, Hong Kong, China
}

(Received 12 June 2000; published 7 March 2001)

\begin{abstract}
The photonic band gap (PBG) effect on the fluorescence lifetime of molecules in three-dimensional colloidal photonic crystals is studied theoretically by means of a full vectorial quantum electrodynamics. In these colloidal systems where the refractive index contrast is as low as below 1.2, the PBG-induced lifetime variation is found to be below 5\%. Our theoretical simulations can help to distinguish the pure PBG effect on the observed fluorescence lifetime variation from other effects such as complex electronic and chemical interactions that can occur in condensed-matter materials.
\end{abstract}

DOI: 10.1103/PhysRevB.63.125106

PACS number(s): 42.70.Qs, 32.80.-t, 42.50.Dv

\section{INTRODUCTION}

Atomic spontaneous emission can be engineered by modifying the photon density of states (DOS) in mesoscopic structures. ${ }^{1}$ In the last decade, there appeared great interest in controlling the spontaneous emission rate via threedimensional (3D) photonic crystals, ${ }^{2-12}$ which are artificial periodic materials. It was suggested that if the atomic transition frequency lies inside the photonic band gap (PBG), the spontaneous emission will be completely inhibited, ${ }^{2}$ as there is no photon mode that can perturb atomic states.

The variation of atomic/molecular spontaneous emission in photonic crystals has become the subject of extensive experimental observations as well as theoretical discussions. ${ }^{1-9}$ Martorell and Lawandy reported the first observation of inhibited spontaneous emission (SE) of dye molecules in dilute colloidal crystals composed of polystyrene microspheres in water. ${ }^{4}$ They found a single exponential decay of the excited state and a variation of the fluorescence lifetime of 1.75 times. However, Tong et al. argued that this remarkable variation was caused mainly by electronic and chemical interactions between dye molecules and the medium, instead of a pure PBG effect. ${ }^{5}$ Besides these complicated electronic and chemical interactions, other effects such as lattice defects, dislocations, and randomness inherent in the system may also influence the atom-field interaction, and, therefore, the SE. Thus it is very helpful to use accurate theoretical approaches to distinguish the pure PBG effect of an ideal photonic crystal on fluorescence lifetimes from other factors in observations made in a realistic photonic crystal.

Although many theoretical discussions have been reported on this subject in recent years, ${ }^{4,8,11,12}$ so far they either adopted a too simplified an isotropic model that is essentially scalar, ${ }^{11}$ or treated spontaneous emission classically, ${ }^{12}$ or adopted phenomenological models. ${ }^{4,8}$ In highly inhomogeneous media like 3D photonic crystals, electromagnetic (EM) fields are also highly inhomogeneous, therefore, we have to consider photon-atom coupling in a full vectorial way. In a recent work ${ }^{10}$ we developed a method to study quantum optics problems in 3D photonic crystals based on a vectorial QED theory. ${ }^{13}$ This method allows for a quantitative understanding of light-atom interaction, and is best suited for our current task to investigate quantitatively the pure PBG effect on fluorescence lifetime of molecules embedded in colloidal photonic crystals. We will limit our discussion only to several reported experimental systems, where numerical calculation will be evaluated to clarify the true PBG effect. We only focus on what the real PBG effect should be in these experimental systems. Other effects are discussed briefly in a general way.

\section{THEORETICAL FORMALISM}

Conventionally, spontaneous emission in a vacuum can be solved by the Weisskopf-Wigner approximation theory. ${ }^{10,13}$ The excited state decays exponentially to the ground state, with the decay constant being

$$
\Gamma=2 \pi \rho(\omega, \mathbf{r})=2 \pi \sum_{\mathbf{k}}\left|g_{\mathbf{k}}(\mathbf{r})\right|^{2} \delta\left(\omega-\omega_{\mathbf{k}}\right),
$$

where $g_{\mathbf{k}}(\mathbf{r})$ is the transition matrix of the excited state. In the dipole approximation, it reads $g_{\mathbf{k}}(\mathbf{r})=-\mathbf{u} \cdot \mathbf{E}_{\mathbf{k}}(\mathbf{r}) / \hbar$, where $\mathbf{u}=u_{0} \hat{\mathbf{u}}$ is the dipole moment, and $\mathbf{E}_{\mathbf{k}}(\mathbf{r})$ $=\sqrt{\hbar \omega_{k} / 2 \epsilon_{0} V} e^{i \mathbf{k} \cdot \mathbf{r}}$ is the quantized electric field of mode $\mathbf{k}, \mathrm{a}$ plan wave function. Here $V$ is the volume of the system, and $\epsilon_{0}$ is the vacuum permittivity. For a general multilevel atom/ molecule, we can use the perturbation theory and the Fermi's golden rule. The result is uniform to the Weisskopf-Wigner approximate theory, i.e., Eq. (1).

The photon DOS is defined as $\rho(\omega)=\Sigma_{\mathbf{k}} \delta\left(\omega-\omega_{\mathbf{k}}\right)$. For a vacuum, we can easily derive

$$
\begin{gathered}
\rho(\omega)=V \omega^{2} / \pi^{2} c^{3}, \\
\rho(\omega, \mathbf{r})=\omega^{3} u_{0}^{2} / 6 \pi^{2} \epsilon_{0} \hbar c^{3},
\end{gathered}
$$

where we have assumed that the direction of the dipole moment $(\hat{\mathbf{u}})$ is randomly oriented in space, and a directional average is taken. For a homogeneous medium with a refractive index of $n_{0}$, the photon dispersion $\omega_{k}=k c / n_{0}$ means that the volume of phase space below the frequency $\omega$ increases to $n_{0}^{3}$ times that in vacuum, and so does the number of photons. In addition, the quantized electric-field amplitude of the photon $\left(\sqrt{\hbar \omega_{k} / 2 \epsilon_{0} n_{0}^{2} V}\right)$ reduces by a factor of $n_{0}$ 
compared to that in vacuum. Therefore, the DOS and the function $\rho(\omega, \mathbf{r})$ in a homogeneous medium are a cubical and linear functions of the refractive index $n_{0}$, respectively. Similar to Eq. (2), we have

$$
\begin{gathered}
\rho(\omega)=V n_{0}^{3} \omega^{2} / \pi^{2} c^{3}, \\
\rho(\omega, \mathbf{r})=\omega^{3} n_{0} u_{0}^{2} / 6 \pi^{2} \epsilon_{0} \hbar c^{3} .
\end{gathered}
$$

A detailed investigation of the quantum theory of lightatom interaction in a realistic 3D photonic crystal ${ }^{10}$ shows that the Weisskopf-Wigner approximate theory is also applicable even for highly inhomogeneous media, and spontaneous emission follows a simple exponential-decay behavior. The DOS and the function $\rho(\omega, \mathbf{r})$ are now superpositions of Bloch's states, ${ }^{10}$

$$
\rho(\omega)=\sum_{n \mathbf{k}} \delta\left(\omega-\omega_{n \mathbf{k}}\right)=\frac{V}{(2 \pi)^{3}} \sum_{n} \int_{\mathrm{BZ}} d^{3} \mathbf{k} \delta\left(\omega-\omega_{n \mathbf{k}}\right),
$$

$$
\begin{aligned}
\rho(\omega, \mathbf{r})= & \sum_{n \mathbf{k}}\left|g_{n \mathbf{k}}(\mathbf{r})\right|^{2} \delta\left(\omega-\omega_{n \mathbf{k}}\right) \\
= & \frac{u_{0}^{2} c^{2}}{2 \hbar \epsilon_{0}(2 \pi)^{3} \epsilon^{2}(\mathbf{r})} \sum_{n} \int_{\mathrm{BZ}} d^{3} \mathbf{k} \frac{\left|\hat{\mathbf{u}} \cdot\left[\nabla \times \mathbf{H}_{n \mathbf{k}}(\mathbf{r})\right]\right|^{2}}{\omega_{n \mathbf{k}}} \\
& \times \delta\left(\omega-\omega_{n \mathbf{k}}\right) \\
= & \frac{u_{0}^{2} c^{2}}{2 \hbar \epsilon_{0}(2 \pi)^{3} \epsilon^{2}(\mathbf{r})} \sum_{n} \int_{\mathrm{BZ}} d^{3} \mathbf{k} \frac{\left|\nabla \times \mathbf{H}_{n \mathbf{k}}(\mathbf{r})\right|^{2}}{3 \omega_{n \mathbf{k}}} \\
& \times \delta\left(\omega-\omega_{n \mathbf{k}}\right),
\end{aligned}
$$

where BZ denotes the first Brillouin zone of the crystal. In the final equality of Eq. (5) we have assumed that $\hat{\mathbf{u}}$ is randomly oriented in space, and an average over $\hat{\mathbf{u}}$ yields a number of $\frac{1}{3}$. The Bloch's state $|\mathbf{n}, \mathbf{k}\rangle$ at the wave vector of $\mathbf{k}$ and the $n$th photonic band for a periodic structure with the dielectric function of $\epsilon(\mathbf{r})$ can be solved numerically by a plane-wave expansion method. ${ }^{14,15}$ The eigenmode has a frequency of $\omega_{n \mathbf{k}}$ and a magnetic-field function of $\mathbf{H}_{n \mathbf{k}}(\mathbf{r})$, which satisfies the following orthogonalization and normalization relations:

$$
\int_{V} \mathbf{H}_{n^{\prime} \mathbf{k}^{\prime}}^{*}(\mathbf{r}) \cdot \mathbf{H}_{n \mathbf{k}}(\mathbf{r}) d^{3} \mathbf{r}=V \delta_{n, n^{\prime}} \delta\left(\mathbf{k}-\mathbf{k}^{\prime}\right) .
$$

Equation (6) is automatically satisfied in our numerical scheme that uses the wave propagation equation for the magnetic field, ${ }^{14,15}$ as here the eigenvectors obtained by standard matrix-diagonalization techniques are normalized with a norm of unity.

The function $\rho(\omega, \mathbf{r})$ defined in Eqs. (2), (3), and (5) consists of a contribution of the atomic dipole $\mathbf{u}$, which is a natural reflection of light-atom interaction. If the dipole moment is randomly oriented in space, this function reduces to the local density of states defined in Refs. 13,16, and 17, apart from a proportional constant $u_{0}^{2} \omega / 6 \hbar \epsilon_{0}$.
For a 3D photonic crystal, numerical calculation is necessary to obtain the DOS and $\rho(\omega, \mathbf{r})$ from Eqs. (4) and (5), as no analytical solution is available. The full vectorial treatment is quite complex and tedious; it involves several numerical steps: discretizing the Brillouin zone into many sampling cells of the Bloch wave vector, solving the photonic bands and EM fields at each cell by the plane-wave expansion method, and finally calculating the DOS and $\rho(\omega, \mathbf{r})$ by summing the contribution from all the eigenmodes via Eqs. (4) and (5). Because of the crystalline symmetry, it is enough to only consider an irreducible BZ. To improve the numerical accuracy, in our calculations we employ a modified tetrahedral integration method ${ }^{18}$ combined with an linear interpolation technique, ${ }^{19}$ which have been readily developed in electronic systems. The tetrahedral integration method correctly accounts for the integration weight of the discretizing points around the boundary of the irreducible BZ. The interpolation technique enables us to obtain quite stable numerical results using rather a small number of discretization cells in the BZ, and greatly releases the computational efforts.

\section{RESULTS AND DISCUSSIONS}

All the experimental observations concerned the fluorescence lifetime of dye molecules in colloidal systems. ${ }^{4-9}$ The observed lifetime is generally composed of two parts: one is due to the spontaneous emission of electronic excited states, the other is caused by other nonradiative internal processes in molecules. The corresponding decay rates can be denoted as $\Gamma=1 / \tau_{r}$ and $1 / \tau_{n r}$, respectively. The total fluorescence lifetime $\tau$ is given by $1 / \tau=1 / \tau_{r}+1 / \tau_{n r}$. The PBG effect only contributes to the variation of radiative lifetime $\tau_{r}$, and can be observed when quantum efficiency of the molecules is high, namely, $\tau_{r} \ll \tau_{n r}$ and $\tau \approx \tau_{r}$.

The spontaneous emission rate for general molecules in any photonic crystal systems can be calculated via Eq. (1): $\Gamma=2 \pi \rho(\omega, \mathbf{r})$. The function $\rho(\omega, \mathbf{r})$ given by Eq. (5), can be decomposed into a product of two parts as

$$
\rho(\omega, \mathbf{r})=\left[u_{0}^{2}(\omega, \mathbf{r}) / 3 \hbar^{2}\right]\left[\left(\hbar \omega / 2 \epsilon_{0}\right) \rho_{c}(\omega, \mathbf{r})\right],
$$

with the former factor reflecting the atomic states; the latter is only related to the macroscopic structure for EM waves, and $\rho_{c}$ denotes the local density of states. ${ }^{16,17}$ Generally, the dipole moment $u_{0}$ is determined by the electronic structures of molecules, which is subject to the influence of local electronic or chemical interactions with a microscopic background. Thus it becomes a function of the emission photon frequency as well as the molecular position. In addition, as the fluorescence might correspond to a transition among many degenerate energy levels of dye molecules, the dipole moment is the sum of all possible transitions from excited states to lower states via emission of a photon of frequency $\omega$. The separation of contributions from the electronic/ chemical interactions (molecules) and the photonic interaction (photonic crystals) in Eq. (7) enables us to distinguish the PBG effect on the observed fluorescence variation in a quantitative way.

Below, we only discuss some of the previous experiments, and make a comparison with our theoretical calcula- 


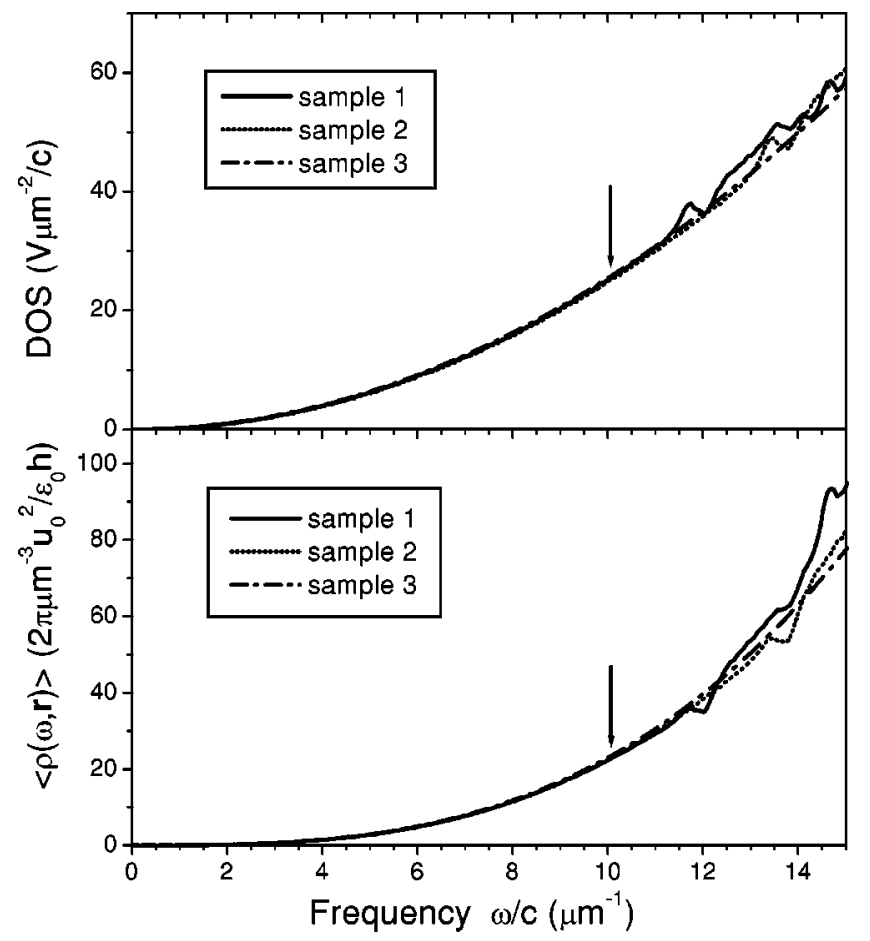

FIG. 1. DOS and averaged $\rho(\omega, \mathbf{r})$ plots for three systems: colloidal crystals of polystyrene spheres in water with parameters of $a=0.338 \mu \mathrm{m}, f=0.101$ (sample 1), $a=0.296 \mu \mathrm{m}, f=0.082$ (sample 2), and colloidal liquid with $f=0.101$ (sample 3). Some symbols in the coordinate labels are $\epsilon_{0}$, the permittivity of vacuum; $h$, the Planck constant; $V$, the volume of the photonic crystal; $u_{0}$, the dipole moment; and $c$, the light speed in vacuum.

tions in regard to the $\mathrm{PBG}$ effect, which allows one to judge whether the observed variation in the fluorescence lifetime could be attributed purely to the PBG effect. In the experiment by Martorell and Lawandy, ${ }^{4}$ fluorescence lifetimes of Kiton Red in three colloidal samples were measured: Samples 1 and 2 are both face-centered-cubic (fcc) lattice of polystyrene microspheres $(n=1.6)$ in water $(n=1.33)$. The filling fraction and lattice constant are $f=0.101, a$ $=0.338 \mu \mathrm{m}$, and $f=0.082, a=0.296 \mu \mathrm{m}$ for samples 1 and 2, respectively. Sample 3 is a disordered colloidal medium with $f=0.101$. The measured lifetimes for the three samples are $\tau_{1}=1.75 \tau_{2}=1.75 \tau_{3}$, and this significant variation was attributed to the inhibition of spontaneous emission, a pure PBG effect. However, later investigations in similar systems by Tong et al. ${ }^{5}$ and Megens et al. ${ }^{8}$ showed that electronic interaction between a dye molecule and a medium might account for a large fraction of the lifetime change observed. The controversy has continued until now. ${ }^{9}$

To verify to what extent the PBG effect is pure, we calculate the DOS and the function $\rho(\omega, \mathbf{r})$ for the three samples. The DOS and the average of $\rho(\omega, \mathbf{r})$ over different molecular positions, denoted as $\langle\rho(\omega, \mathbf{r})\rangle$, are plotted in Fig. 1. The curves for colloidal crystals are obtained according to Eqs. (3) and (4) by solving over 2300 points in the irreducible BZ with the use of 343 plane-wave expansions. As the refractive index contrast is quite small, the numerical convergence is quite satisfactory. The molecule moment $\mathbf{u}$ is

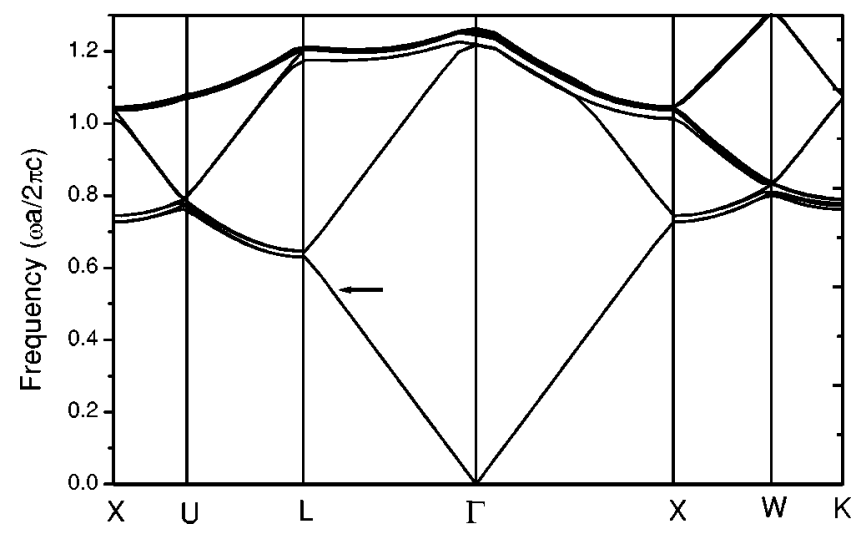

FIG. 2. Photonic band structures of the colloidal crystal corresponding to sample 1 in Fig. 1.

assumed to be randomly oriented, and remains unchanged as a constant. The general variance of $\rho(\omega, \mathbf{r})$ is below $7 \%$ of the averaged value, indicating that the radiative lifetime is slightly dependent on the molecule position. For the disordered colloidal sample, we adopt the vectorial effective medium theory-the Maxwell-Garnett theory, $\left(\bar{\epsilon}-\epsilon_{2}\right) /(\bar{\epsilon}$ $\left.+2 \epsilon_{2}\right)=f\left(\epsilon_{1}-\epsilon_{2}\right) /\left(\epsilon_{1}+2 \epsilon_{2}\right)$ - then use Eq. (3), where $n_{0}$ $=\sqrt{\bar{\epsilon}}$. We also use the scalar effective-medium theory, $\bar{\epsilon}$ $=f \epsilon_{1}+(1-f) \epsilon_{2}$, and find that the scalar $n_{0}$ is about $0.2 \%$ larger than the vectorial value. The excellent agreement is due to the small dielectric contrast. There appears a shallow dip in the DOS and $\langle\rho(\omega, \mathbf{r})\rangle$ centered at a wavelength $\lambda$ of about 524 and $455 \mathrm{~nm}$ (corresponding to a frequency $\omega / c$ of 12.0 and $13.8 \mu \mathrm{m}^{-1}$ ) for samples 1 and 2, respectively. Here $c$ is the light speed in vacuum. To understand better, we also plot the photonic band structures of colloidal crystals in Fig. 2 for sample 1, where the frequency $\omega / c$ is in units of 18.6 $\mu \mathrm{m}^{-1}$. Almost the same band structures are found for sample 2. It is seen that the dip corresponds exactly to the narrow directional band gap (width below $3 \%$ ) between 2 and 3 bands along the (111) ( $L$-point) direction. The maximum variation in $\langle\rho(\omega, \mathbf{r})\rangle$ (and thus the lifetime $\tau_{r}$ ) is about $5 \%$ in the dip.

The frequency of probe light $(\lambda=625 \mathrm{~nm}$, or equivalently $\omega / c=10.1 \mathrm{\mu m}^{-1}$, shown by the arrows) is well below this dip for both samples 1 and 2, and enters the longwavelength regime, where the photon dispersion is almost linear. Then it is evident that no apparent PBG effect can be observed in the fluorescence lifetime variation. In fact, the values of $\langle\rho(\omega, \mathbf{r})\rangle$ for the two crystals differ from each other below $2 \%$, and they are about $4 \%$ smaller than for the liquid. Therefore, the PBG-induced lifetime variation in the three samples should be less than $5 \%$, far below the observed variation of 1.75 times. This weak PBG effect persists even if the probe light frequency falls right inside the directional gap. The observed significant variation can only be attributed to different weight of contributions from electronic and chemical interactions in three samples, which in turn might modify the dipole moment $u_{0}(\omega, \mathbf{r})$, as well as $\tau_{n r}$, differently.

Following this scheme, we further analyze two recent experiments where significant disagreement also arose. ${ }^{7-9}$ 


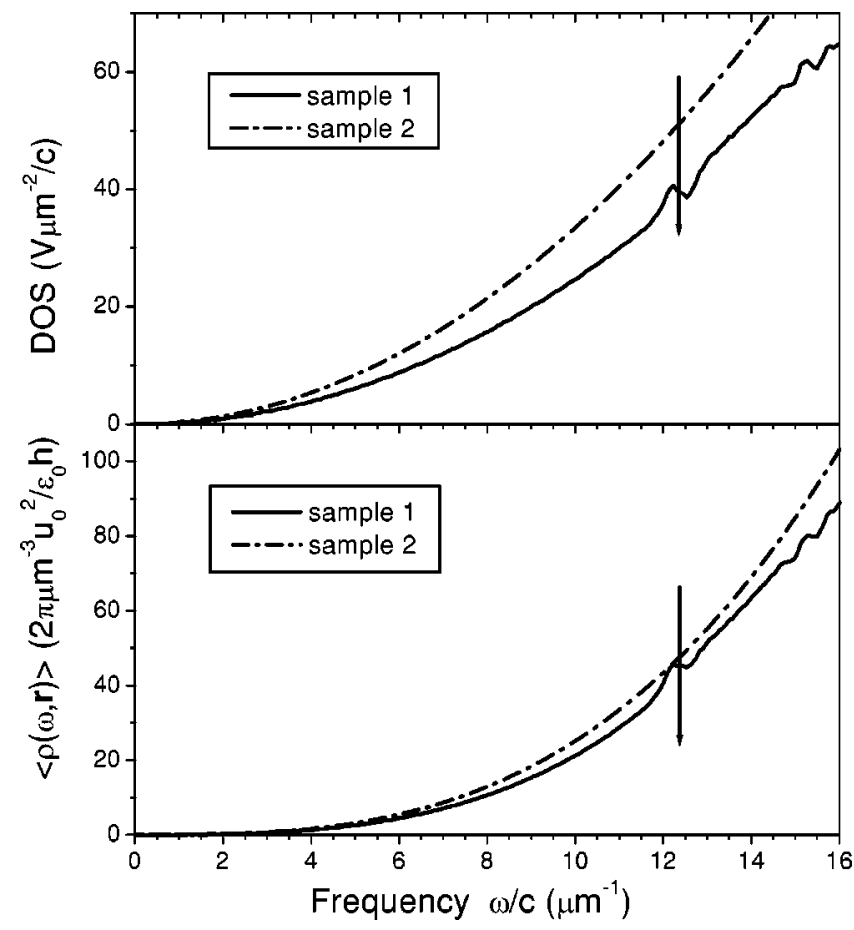

FIG. 3. DOS and averaged $\rho(\omega, \mathbf{r})$ plots for an artificial closepacked opal filled with a polymer (sample 1), and a homogeneous polymer medium (sample 2 ).

Petrov et al. measured the fluorescence of dye molecules embedded in artificial close-packed opal (silica, $n=1.3$ ) filled with a polymer $(n=1.49)$, and observed spontaneous decay kinetics containing both accelerated and inhibited exponential-decay components compared to the fluorescence in a reference polymer matrix. They attributed the lifetime variation to the redistribution of photon DOS in photonic crystals, also a pure photonic effect. We have calculated photonic band structures for this opal, and found nearly the same characteristics as in Fig. 2. We also calculate the DOS and $\langle\rho(\omega, \mathbf{r})\rangle$ for the opal (sample 1), and reference polymer matrix (sample 2), which are shown in Fig. 3. The variance of $\rho(\omega, \mathbf{r})$ is below $8 \%$ of the averaged value, and thus the position dependence is also weak. The DOS and $\langle\rho(\omega, \mathbf{r})\rangle$ for a polymer are higher than for the opal, which is clear from Fig. 3, and also from Eq. (2). At the frequency of probe light $\left(\lambda=510 \mathrm{~nm}\right.$, or $\omega / c=12.3 \mu \mathrm{m}^{-1}$, shown by arrows), we have found a ratio of about 1.3 for the DOS and 1.05 for $\langle\rho(\omega, \mathbf{r})\rangle$ between samples 2 and 1 . This means that the photonic-induced fluorescence lifetime in opal crystal should be close to that in the polymer matrix. However, in the experiment, $\tau \approx 6.5 \mathrm{~ns}$ (accelerated) and $\tau \approx 11.6 \mathrm{~ns}$ (inhibited) were observed, compared to $\tau \approx 9.5 \mathrm{~ns}$ for the polymer matrix, in great contradiction with the theoretical prediction. The most apparent variation in lifetime is caused by a directional weak gap, corresponding to a dip in the DOS and $\langle\rho(\omega, \mathbf{r})\rangle$ (centered at about $506 \mathrm{~nm}$, or $\omega / c=12.4 \mu \mathrm{m}^{-1}$ ) for the opal crystal. The PBG effect is about $2 \%$ at the probe light frequency, and the maximum value is also below $5 \%$. Thus the observed significant variation of the fluorescence lifetime is by no means caused by PBG effects, but instead

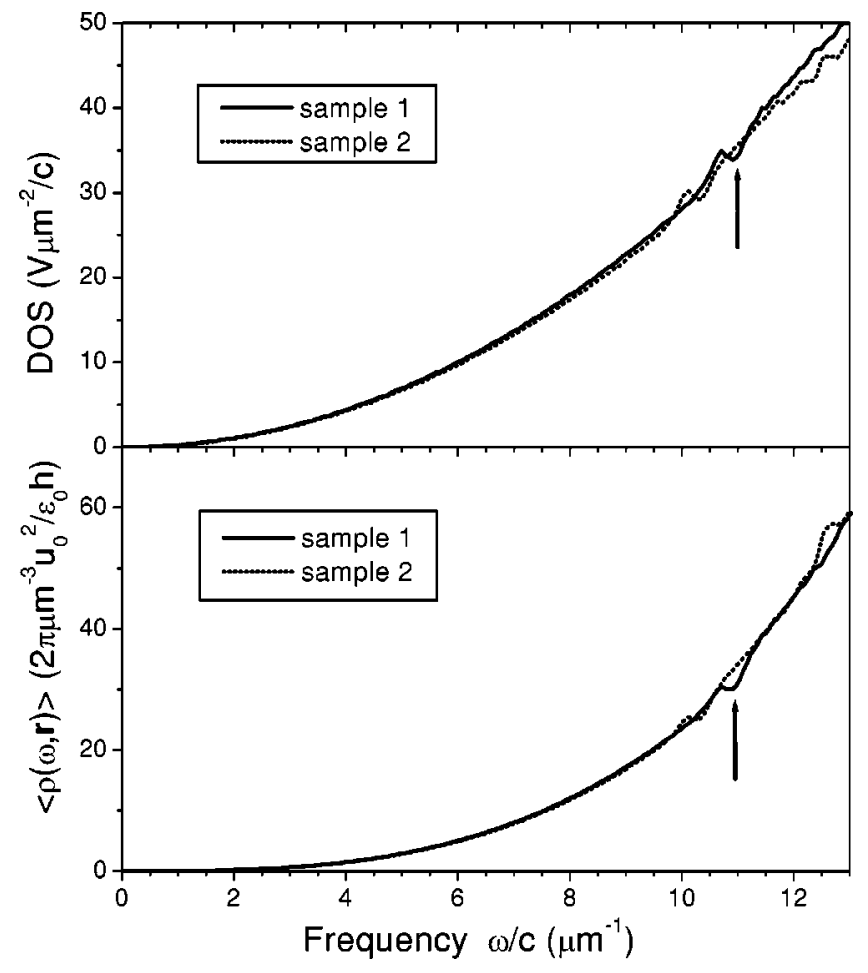

FIG. 4. DOS and averaged $\rho(\omega, \mathbf{r})$ plots for two colloidal crystals of silica microspheres in water with parameters of $a$ $=0.3574 \mu \mathrm{m}, f=0.65$ (sample 1) and $a=0.3826 \mu \mathrm{m}, f=0.53$ (sample 2).

more likely caused by electronic/chemical interactions, which differ much between the polymer reference, a bulk material, and the submicrometer-size inverse-opal structure. ${ }^{5}$ The two fluorescence components observed in the inverseopal structure can only be ascribed to two $u_{0}(\omega, \mathbf{r})$ and $\tau_{n r}$ values, which should be caused by two overwhelming types of microscopic background inside the submicrometer-sized polymer.

Another observation for dye molecule embedded inside silica microspheres $(n=1.45)$, forming a fcc lattice in water $(n=1.33)$, showed no appreciable PBG effect. ${ }^{8}$ We have considered two opal crystals with parameters of $a$ $=0.3574 \mu \mathrm{m}, f=0.65$ (sample 1) and $a=0.3826 \mu \mathrm{m}, f$ $=0.53$ (sample 2). The DOS and $\langle\rho(\omega, \mathbf{r})\rangle$ are displayed in Fig. 4. The variance of $\rho(\omega, \mathbf{r})$ is below $3 \%$ of the averaged value. The frequency of the probe light $(\lambda=577 \mathrm{~nm}, \omega / c$ $\left.=10.9 \mu \mathrm{m}^{-1}\right)$ lies inside the DOS dip of sample 1. At this frequency, the calculated value of $\langle\rho(\omega, \mathbf{r})\rangle$ for sample 2 is about $9 \%$ larger than that for sample 1 . The center of the dip in $\langle\rho(\omega, \mathbf{r})\rangle$ for sample 2 lies at $\lambda=610 \mathrm{~nm}(\omega / c$ $\left.=10.3 \mu \mathrm{m}^{-1}\right)$; at this frequency, the value of $\langle\rho(\omega, \mathbf{r})\rangle$ for sample 1 is about $4 \%$ larger than that for sample 2 because of the PBG-effect. Accordingly, the PBG-induced variation in $\langle\rho(\omega, \mathbf{r})\rangle$ for each sample can be set to be about $4 \%$, below $5 \%$.

Beyond the two dips, $\langle\rho(\omega, \mathbf{r})\rangle$ for the two crystals differs very little (below $2 \%$ ) in the wide frequency range below $\lambda=520 \mathrm{~nm}\left(\omega / c=12.1 \mu \mathrm{m}^{-1}\right)$; this is in good agreement with the experimental observations, where the fluorescence 
lifetime for the two crystals differs by less than $3 \% .{ }^{8}$ On the other hand, the observed lifetime has a tiny change in the range of $540-700 \mathrm{~nm}\left(9.0-11.6 \mu \mathrm{m}^{-1}\right)$; however, the photonic-induced lifetime due to the PBG effect should increase nearly cubically with respect to the wavelength, according to Fig. 4. It has been suggested that this discrepancy could be caused by nonradiative decay, which is fast at long wavelengths. ${ }^{8}$ However, as the quantum efficiencies of dye molecules are high, it is also likely that the appreciable change of dipole moment $u_{0}(\omega, \mathbf{r})$ with wavelength plays the key role. As the lifetime is an inverse square function of $u_{0}$ [see Eq. (5)], the increase of $u_{0}$ at long wavelengths, for example $u_{0} \sim \lambda^{3 / 2}$, can well compensate for the effect due to the change of $\rho(\omega, \mathbf{r})$.

In the above discussions we only considered perfect photonic crystal structrues. In a realistic photonic crystal structure, there always exist many kinds of randomness, such as the dispersion in particle size, lattice defects and dislocations. The presence of disorder will introduce resonant or localized states into the band gap, ${ }^{20}$ and thus weaken the dip in the DOS curves. Such states usually possess larger spatial fluctuations in the EM fields, and, therefore, can enhance the atom-field interaction. This is especially true in the case of structures with a complete band gap, where the localization of EM fields is very strong. ${ }^{20}$ In these structures, the fluorescence lifetime varies (fluctuates) much with respect to molecules positions. However, in colloidal structures where the refractive index is as small as below 1.2, we expect that such disorder-induced spatial fluctuation is weak, and the fluctuation of the fluorescence lifetime is also small. In the limit of strong disorders, i.e., a colloidal liquid, no PBG effect was observed, ${ }^{4,8}$ as expected (for a homogeneous medium). So we believe that the major influence on the molecular fluorescence lifetime comes from the electronic/chemical interactions, instead of a PBG effect, even in a realistic sample.

\section{CONCLUSIONS}

In summary, we have employed a vectorial QED approach to investigate the PBG effect on molecular fluorescence lifetime in 3D colloidal photonic crystals, and found that the pure PBG effect on the fluorescence lifetime is below $5 \%$ for these systems with a small dielectric contrast. In addition, the fluorescence lifetime can be appreciably influenced by electronic and chemical interactions of molecules with their microscopic environment. The disagreement between accurate theoretical calculations and experimental observations clearly implies that the remarkable effect of electronic/chemical interactions on the molecular fluorescence lifetime remained residual in most previous experiments in colloidal systems. The theoretical simulations can be of great help in quantitatively distinguishing the contribution of PBG effects on observed variation of fluorescence lifetimes from other complex electronic and chemical interactions.

\section{ACKNOWLEDGMENT}

This work was supported by Hong Kong RGC Grant No. HKUST 6160/99P.
${ }^{1}$ E. M. Purcell, Phys. Rev. 69, 681 (1946).

${ }^{2}$ E. Yablonovitch, Phys. Rev. Lett. 58, 2059 (1987).

${ }^{3}$ J. D. Joannopoulos, R. D. Meade, and J. N. Winn, Photonic Crystals (Princeton University Press, Princeton, 1995).

${ }^{4}$ J. Martorell and N. M. Lawandy, Phys. Rev. Lett. 65, 1877 (1990).

${ }^{5}$ B. Y. Tong, P. K. John, Y. T. Zhu, Y. S. Liu, S. K. Wong, and W. R. Ware, J. Opt. Soc. Am. B 10, 356 (1993).

${ }^{6}$ N. M. Lawandy, J. Opt. Soc. Am. B 10, 2144 (1993).

${ }^{7}$ E. P. Petrov, V. N. Bogomolov, I. I. Kalosha, and S. V. Gaponenko, Phys. Rev. Lett. 81, 77 (1998).

${ }^{8}$ M. Megens, J. E. G. J. Wijnhoven, A. Lagendijk, and W. L. Vos, Phys. Rev. A 59, 4727 (1999).

${ }^{9}$ M. Megens, H. P. Schriemer, A. Lagendijk, and W. L. Vos, Phys. Rev. Lett. 83, 5401 (1999); E. P. Petrov, V. N. Bogomolov, I. I. Kalosha, and S. V. Gaponenko, ibid. 83, 5402 (1999).

${ }^{10}$ Z. Y. Li, L. L. Lin, and Z. Q. Zhang, Phys. Rev. Lett. 84, 4341 (2000)

${ }^{11}$ S. John and J. Wang, Phys. Rev. B 43, 12772 (1991); S. John and T. Quang, Phys. Rev. A 50, 1764 (1994).
${ }^{12}$ T. Suzuki and P. K. L. Yu, J. Opt. Soc. Am. B 12, 570 (1995).

${ }^{13}$ M. O. Scully and M. S. Zubairy, Quantum Optics (Cambridge University Press, Cambridge, 1997); R.J. Glauber and M. Lewenstein, Phys. Rev. A 43, 467 (1991); S. Barnett and R. Loudon, Phys. Rev. Lett. 77, 2444 (1996).

${ }^{14}$ K. M. Ho, C. T. Chan, and C. M. Soukoulis, Phys. Rev. Lett. 65, 3152 (1990).

${ }^{15}$ Z. Y. Li, J. Wang, and B. Y. Gu, Phys. Rev. B 58, 3721 (1998); Z. Y. Li, B. Y. Gu, and G. Z. Yang, Phys. Rev. Lett. 81, 2574 (1998).

${ }^{16}$ K. Busch and S. John, Phys. Rev. E 58, 3896 (1998).

${ }^{17}$ R. Sprik, B. A. van Tiggelen, and A. Lagendijk, Europhys. Lett. 35, 265 (1996).

${ }^{18}$ J. Hanna, M. Watanable, and E. Kato, J. Phys.: Condens. Matter 2, 7445 (1990).

${ }^{19}$ G. Lehmann and M. Taut, Phys. Status Solidi B 54, 469 (1972).

${ }^{20}$ Z. Y. Li, X. Zhang, and Z. Q. Zhang, Phys. Rev. B 61, 15738 (2000); Z. Y. Li and Z. Q. Zhang, ibid. 62, 1516 (2000), and references therein. 